\title{
Immune-checkpoint inhibitors associated with interstitial lung disease in cancer patients
}

\author{
Myriam Delaunay ${ }^{1}, J_{a c q u e s}$ Cadranel $^{2}$, Amélie Lusque $^{3}$, Nicolas Meyer $^{4}$, \\ Valérie Gounant ${ }^{5}$, Denis Moro-Sibilot ${ }^{6}$, Jean-Marie Michot ${ }^{7}$, Judith Raimbourg ${ }^{8}$, \\ Nicolas Girard ${ }^{9}$, Florian Guisier ${ }^{10}$, David Planchard ${ }^{11}$, Anne-Cécile Metivier ${ }^{12}$, \\ Pascale Tomasini ${ }^{13}$, Eric Dansin ${ }^{14}$, Maurice Pérol ${ }^{15}$, Marion Campana ${ }^{16}$, \\ Oliver Gautschi ${ }^{17}$, Martin Früh ${ }^{18}$, Jean-David Fumet ${ }^{19}$, \\ Clarisse Audigier-Valette ${ }^{20}$, Sébastien Couraud ${ }^{21}$, Stéphane Dalle ${ }^{22}$, \\ Marie-Thérèse Leccia ${ }^{23}$, Marion Jaffro ${ }^{24}$, Samia Collot ${ }^{24}$, Grégoire Prévot ${ }^{1}$, \\ Julie Milia ${ }^{1}$ and Julien Mazieres ${ }^{1}$
}

@ERSpublications

Awareness of clinical/radiological presentation of immunotherapy-related pneumonitis is crucial to ensure a diagnosis http://ow.ly/eIMF30bgolf

Cite this article as: Delaunay $\mathrm{M}$, Cadranel J, Lusque A, et al. Immune-checkpoint inhibitors associated with interstitial lung disease in cancer patients. Eur Respir J 2017; 50: 1700050 [https://doi.org/10.1183/ 13993003.00050-2017].

ABSTRACT Immunotherapy is becoming a standard of care for many cancers. Immune-checkpoint inhibitors (ICI) can generate immune-related adverse events. Interstitial lung disease (ILD) has been identified as a rare but potentially severe event.

Between December 2015 and April 2016, we conducted a retrospective study in centres experienced in ICI use. We report the main features of ICI-ILD with a focus on clinical presentation, radiological patterns and therapeutic strategies.

We identified $64(3.5 \%)$ out of 1826 cancer patients with ICI-ILD. Patients mainly received programmed cell death-1 inhibitors. ILD usually occurred in males, and former or current smokers, with a median age of 59 years. We observed $65.6 \%$ grade $2 / 3$ severity, $9.4 \%$ grade 4 severity and $9.4 \%$ fatal ILD. The median (range) time from initiation of immunotherapy to ILD was $2.3(0.2-27.4)$ months. Onset tended to occur earlier in lung cancer versus melanoma: median 2.1 and 5.2 months, respectively ( $\mathrm{p}=0.02)$. Ground-glass opacities (81.3\%) were the predominant lesions, followed by consolidations (53.1\%). Organising pneumonia (23.4\%) and hypersensitivity pneumonitis (15.6\%) were the most common patterns. Overall survival at 6 months was $58.1 \%$ (95\% CI $37.7-73.8 \%)$.

ICI-ILD often occurs early and displays suggestive radiological features. As there is no clearly identified risk factor, oncologists need to diagnose and adequately treat this adverse event.

This article has been amended according to the erratum published in the November 2017 issue of the European Respiratory Journal.

This article has supplementary material available from erj.ersjournals.com

Received: Jan 102017 | Accepted after revision: April 202017

Conflict of interest: Disclosures can be found alongside this article at erj.ersjournals.com

Copyright @ERS 2017 
Affiliations: 'Service de Pneumologie, Hôpital Larrey, Centre Hospitalier Universitaire, Université Paul Sabatier, Toulouse, France. ${ }^{2}$ Service de Pneumologie, APHP Hôpital Tenon and Sorbonne Universités, UPMC Univ Paris 06, Paris, France. ${ }^{3}$ Cellule Biostatistique, Bureau des Essais Cliniques, Institut Claudius Regaud, IUCT-0, Toulouse, France. ${ }^{4}$ Service de Dermatologie, IUCT, Centre Hospitalier Universitaire, Université Paul Sabatier, Toulouse, France. ${ }^{5}$ Service d'Oncologie Thoracique, University Hospital Bichat, APHP, Paris, France. ${ }^{6}$ Department of Thoracic Oncology, Centre Hospitalier Universitaire, Grenoble, France. ${ }^{7}$ Hematological Malignancies and Drug Development Departments, Gustave Roussy, Cancer Campus Grand Paris, Villejuif, France. ${ }^{8}$ Service d'Oncologie Médicale, Institut de Cancérologie de l'Ouest, St Herblain, Inserm U892, Nantes, France. 'Service de Pneumologie, Institut de Cancérologie des Hospices Civils de Lyon, Lyon, France. ${ }^{10}$ Service de Pneumologie, Oncologie Thoracique et Soins Intensifs Respiratoires, Rouen University, Hopital, CIC INSERM 1404, Quant IF-LITIS, Rouen, France. ${ }^{11}$ Medical Oncology Department, Gustave Roussy Cancer Center, Villejuif, France. ${ }^{12}$ Service de Pneumologie, Hôpital Foch, Suresne, France. ${ }^{13}$ Multidisciplinary Oncology and Therapeutic Innovations Dept, Aix Marseille University, Assistance Publique Hôpitaux de Marseille, Marseille, France. ${ }^{14}$ Département de Cancérologie Générale, Centre Oscar Lambret, Lille, France. ${ }^{15}$ Département de Cancérologie Médicale, Centre Léon Bérard, Lyon, France. ${ }^{16}$ Service de Pneumologie, CHRU Tours, Tours, France. ${ }^{17}$ Medical Oncology Department, Lucerne Cantonal Hospital, Luzern, Switzerland. ${ }^{18}$ Medical Oncology Department, Kantonsspital St Gallen, St Gallen, Switzerland. ${ }^{19}$ Département Oncologie Medicale, Centre Georges-Francois Leclerc, Dijon, France. ${ }^{20}$ Service de Pneumonologie, Hôpital Saint Musse, Toulon, France. ${ }^{21}$ Service de Pneumologie Aiguë Spécialisée et Cancérologie Thoracique, Hospices Civils de Lyon, Université Claude Bernard, Lyon, France. ${ }^{22}$ Service de Dermatologie, Hospices Civils de Lyon, Université Claude Bernard, Lyon, France. ${ }^{23}$ Service de Dermatologie, CHU de Grenoble site Nord Hôpital Albert Michallon, Grenoble, France. ${ }^{24}$ Service de Radiologie, Centre Hospitalier Universitaire, Université Paul Sabatier, Toulouse, France.

Correspondence: Julien Mazières, Thoracic Oncology Unit, Respiratory Disease Dept, Hôpital Larrey, CHU Toulouse, Chemin de Pouvourville, 31059 Toulouse Cedex, France. E-mail: mazieres.jachu-toulouse.fr

\section{Introduction}

Immune-checkpoint inhibitors (ICI) represent the latest major breakthrough in oncology and offer a new paradigm for the treatment of different types of advanced solid tumours. Ipilimumab, which inhibits the cytotoxic T-lymphocyte antigen (CTLA)-4 pathway, was the first immune checkpoint blocking antibody to be licensed for advanced melanoma. The identification of the programmed cell-death ligand (PD-L) 1 as a distal immune modulator was at the origin of the development of molecules that targeted PD-1 (nivolumab and pembrolizumab) or PD-L1 (atezolizumab and durvalumab).

Clinical trials have demonstrated the superiority of ICI over standard care for untreated melanoma, pretreated nonsmall cell lung cancer (NSCLC) and, more recently, untreated NSCLC selected according to PD-L1 expression [1-3]. In addition, the benefit of these drugs was recently demonstrated for bladder cancer, head and neck tumours and lymphoma [4-6]. As a consequence, the numbers of patients exposed to these new treatments will increase rapidly, making possible toxicity a major concern.

Overall, ICI present a favourable overall benefit-to-risk profile. However, they are associated with a unique set of toxicities due to their mechanisms of action, termed immune-related adverse events, which are very different from toxicities observed with conventional cytotoxic chemotherapy or targeted therapies. It is important for all clinicians who prescribe immunotherapy to recognise these early on, to clinically assess the effects and to manage the immune-mediated effects, as they can lead to severe toxicity or even death. Pulmonary toxicity has been reported, especially with drugs targeting the PD-1/PD-L1 axis, including some cases of fatal pneumonitis [7-9]. In a recent meta-analysis, the overall incidence of pneumonitis was estimated at $2.7 \%$ for all grades and $0.8 \%$ for grade 3 or higher [10]. In two recent studies, the incidence was even higher (5\% and $11.8 \%)$ [10, 11$]$.

The underlying pathogenesis is partially understood, but the radiological pattern and analyses of bronchoalveolar lavage (BAL) are rarely detailed. Herein, we have collected data from a large series of patients with interstitial lung disease (ILD) that developed during ICI treatment (ICI-ILD) for various cancers. We describe the clinical and radiological presentations with central and multidisciplinary reviewing of all cases, and detail the management of this toxicity and the global outcomes.

\section{Patients and methods}

\section{Study design}

Between December 2015 and April 2016 we enrolled patients treated with ICI in clinical trials, expanded access programmes or after obtaining approval in France and Switzerland. We contacted the main investigators in large centres that are known to treat patients in clinical trials and obtain early access to new drugs. Inclusion criteria were patients aged $>18$ years, with any cancer treated with ICI (CTLA-4, PD-1 and 
PD-L1 inhibitors) and who had developed ILD which was considered by the investigators to be possibly associated with the immunotherapy and that had related data (clinical data, computed tomography (CT) scan and follow-up). The primary objective was the clinical and radiological description of ICI-ILD in cancer patients, after a central review. Secondary objectives were the reporting of overall survival following this adverse event and analysis of therapeutic strategies. The exploratory objective was the analysis of potential underlying mechanisms for ILD based on histological or BAL findings, when available.

\section{Data collection and ethical considerations}

Anonymised clinical data (detailed later) were collected using a standardised datasheet. All participating investigators were trained in good clinical practice and had experience with immunotherapy treatment. All data were collected centrally at the University of Toulouse (Toulouse, France). The data cut-off point was April 30, 2016. The study was conducted in accordance with French law and was approved by a national ethics committee (CEPRO 2016-022).

\section{Definition of cases}

The diagnostic criteria for ICI-ILD were as follows. 1) Correct identification of the drug; 2) singularity of the drug; 3) temporal eligibility; 4) clinical, imaging and pathological pattern of lung involvement conforming to earlier observations with the drug; and 5) exclusion of other causes of ILD [12, 13]. The term often used in the literature is pneumonitis, but we chose ILD instead, because this term better covers the heterogeneity of the different patterns found. Names of all other drugs given and associated with ICIs were collected for each patient and their potential lung toxicities were checked in the Pneumotox database (www.pneumotox.com). All data were reviewed centrally by a panel of pulmonologists specialising in interstitial pneumonitis and oncology.

Severity of ILD was defined according to the Common Terminology Criteria for Adverse Events, version 4.0 [14].

The following characteristics were collected for each patient before ICI exposure: age, sex, smoking habit, performance status, comorbidities (especially prior lung diseases and immunodeficiency), type of cancer, prior chemotherapy regimens, duration of treatment with ICI and other concomitant therapies. In addition, results of bronchial biopsies and BAL (by cytopathology analyses and immunocytochemistry staining of $\mathrm{CD} 3 / \mathrm{CD} 4 / \mathrm{CD} 8)$ were collected. PD-L1 expression was analysed using immunochemistry following local laboratory procedures.

\section{Radiology}

A high-resolution CT (HRCT) scan was mandatory for all patients. All HRCT scans were reviewed centrally by a panel of two radiologists experienced in interstitial lung diseases (Samia Collot and Marion Jaffro), a pulmonologist specialising in interstitial pneumonitis (Grégorie Prévot) and two pulmonologists specialising in oncology. The multidisciplinary panel systematically analysed 15 criteria (online supplementary table S2) and ILD was defined by the occurrence of new and diffuse lung parenchymal abnormalities, including ground-glass opacities, consolidations, interlobular septal thickening and intralobular lines, micronodules, bronchiectasis and architectural distortion on sequential CT scan examinations, according to the American Thoracic Society/European Respiratory Society 2002 classification $[15,16]$.

The interpreters assessed systematically the presence, distribution and extent of each ILD feature on HRCT scans. When possible, a suggestive radiology pattern was provided, i.e. organising pneumonia, nonspecific interstitial pneumonia (NSIP), hypersensitivity pneumonitis, bronchiolitis or unclassifiable, as described previously [15]. All patients had a basal CT scan before starting immunotherapy. We excluded lesions in the target volumes of radiotherapy, lesions caused by cancer progression and any pre-existing lesions caused by chronic obstructive pulmonary disease (COPD), emphysema or prior ILD from the analyses.

\section{Statistics}

The data are expressed as $\mathrm{n}$ (\%) for categorical variables and as median (range) for continuous variables. The Chi-squared or Fisher's exact test was used to compare categorical variables, and the Kruskal-Wallis test was used for continuous variables. Overall survival was defined as the time from diagnosis of pneumonitis until death from any cause or the last follow-up (censored data), and was estimated using the Kaplan-Meier method with 95\% confidence intervals. All reported p-values were two-sided. For all tests, a 
statistical difference was considered significant at the 5\% level. Statistical analyses were conducted using Stata (version 13; StataCorp, College Station, TX, USA).

\section{Results}

\section{Patient characteristics}

We analysed 71 cases and after central review (which included clinical, radiological and microbiological data) we excluded seven cases from the analyses: three cases were considered to be infectious pneumonitis and four cases were tumour progression. 64 cases of ILD were included in our analyses (figure 1). Estimation of overall incidence was 3.5\%: 1826 patients received anti-PD1 from all centres, based on the reported incidence from each centre (64 out of 1826). Patient demographics are shown in table 1.

Of the 64 included cases, 48 (75\%) had NSCLC (52.1\% adenocarcinomas, 33.3\% squamous-cell carcinomas and $14.6 \%$ large-cell carcinomas or not otherwise specified), 13 (20.3\%) had melanomas and three $(4.7 \%)$ patients had other types of cancer (nasopharyngeal carcinoma $n=2$ and Hodgkin lymphoma $\mathrm{n}=1)$. Four (6.9\%) patients received anti-CTLA-4, 46 patients received anti-PD1 (79.3\%) and eight $(13.8 \%)$ patients received anti PD-L1; none received a combination of immunotherapy. We did not know whether six patients had received anti-CTLA-4 or anti-PD1/PD-L1 because they were enrolled in a double-blind trial. Most patients were male (84.4\%) and current $(26.7 \%)$ or ex-smokers (53.3\%), with a median consumption of 40 (5-80) pack-years, and a median age of 59 years. 17 (27.4\%) patients had a medical history of COPD or emphysema.

One patient had idiopathic pulmonary fibrosis and one had untreated granulomatosis with polyangiitis disease; both diseases are usually considered as a contraindication for ICI. These two cases were included because clinical radiological data and time to ILD onset conformed to our ICI-ILD criteria and did not favour idiopathic pulmonary fibrosis or granulomatosis exacerbation. The patient who had idiopathic pulmonary fibrosis received two infusions of anti-PD1; the time to ILD was 3 weeks. For the patient with granulomatosis with polyangitis, the time until ILD onset was 10 weeks and he received four infusions. These two cases showed rapid improvement after steroid treatment. No other autoimmune diseases were reported.

Most patients had stage IV disease when immunotherapy was introduced with frequent lung metastases (58.5\% for NSCLC and $66.7 \%$ for melanoma) and lymph node metastasis (51.2\% for NSCLC and $36.4 \%$ for melanoma). Most patients (87.5\%) had received at least one previous line of treatment before immunotherapy, especially a platinum-based doublet, an epidermal growth factor receptor (EGFR) inhibitor for NSCLC or a BRAF-inhibitor for melanoma (25\% had received one line of treatment, $32.8 \%$ had received two lines and $29.7 \%$ had received more than three lines). Median duration between a previous treatment line and ICI was 48 days (0.0-36.0 months).

At the time of ILD onset, the best objective tumour response rate until ICI was $35.9 \%$ (23 out of 64), according to RECIST (Response Evaluation Criteria in Solid Tumors 1.1) (one complete response and 22 partial responses) [17]. The objective tumour response rates were $33.3 \%$ and $46.2 \%$ for NSCLC and

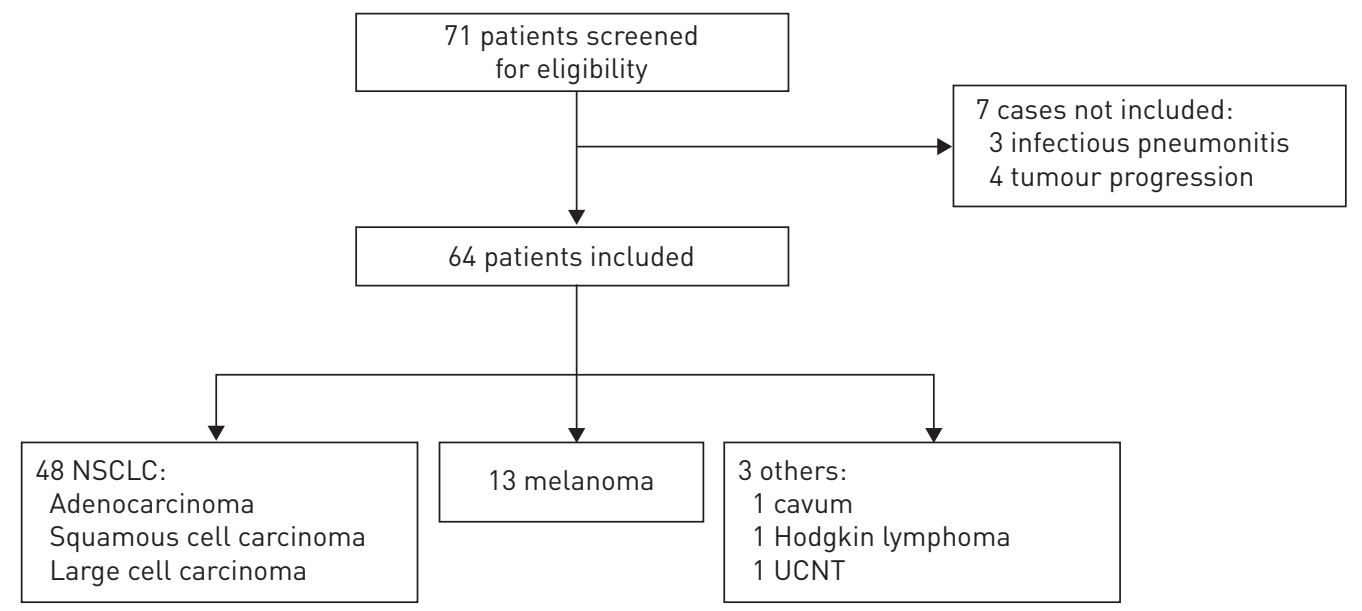

FIGURE 1 Study flowchart. NSCLC: nonsmall cell lung cancer; UCNT: undifferentiated carcinoma of nasopharyngeal type. 
TABLE 1 Baseline characteristics of the study population

\begin{tabular}{|c|c|c|}
\hline & Patients & Frequency \\
\hline Sex & 64 & \\
\hline Male & & $54(84.4)$ \\
\hline Female & & $10(15.6)$ \\
\hline Age at diagnosis years & 63 & 59 (22-83) \\
\hline$<60$ & & $33(52.4)$ \\
\hline$\geqslant 60$ & & $30(47.6)$ \\
\hline Unknown & & 1 \\
\hline Smoking history & 60 & \\
\hline Never-smoker & & $12(20.0)$ \\
\hline Current smoker & & $16(26.7)$ \\
\hline Ex-smoker & & $32(53.3)$ \\
\hline Unknown & & 4 \\
\hline Pack-years & & $40(5-80)$ \\
\hline Medical history & 62 & \\
\hline Chronic infection & & $1(1.6)$ \\
\hline Immunodeficiency & & $1(1.6)$ \\
\hline Other type of cancer & & $11(17.7)$ \\
\hline COPD & & $12(19.4)$ \\
\hline Emphysema & & $6(9.7)$ \\
\hline Pulmonary fibrosis & & $1(1.6)$ \\
\hline ECOG performance status & 64 & \\
\hline $0-1$ & & 59 (92.2) \\
\hline $2-3$ & & $5(7.8)$ \\
\hline Diagnosis of tumour & 64 & \\
\hline Lung cancer & & $48(75.0)$ \\
\hline Adenocarcinoma & & $25(52.1)$ \\
\hline Squamous & & 16 (33.3) \\
\hline Other & & $7(14.6)$ \\
\hline Melanoma & & 13 (20.3) \\
\hline Other & & $3(4.7)$ \\
\hline PD-L1/PD1 status & 15 & \\
\hline Positive & & 12 (80.0) \\
\hline Negative & & $3(20.0)$ \\
\hline Unknown/not assessed & & 49 \\
\hline Metastatic site & 55 & \\
\hline Pulmonary metastasis & & $32(58.2)$ \\
\hline Lung cancer & 48 & $24(58.5)$ \\
\hline Melanoma & 13 & $8(66.7)$ \\
\hline Lymph nodes & & $26(47.3)$ \\
\hline Lung cancer & 48 & $21(51.2)$ \\
\hline Melanoma & 13 & $4(36.4)$ \\
\hline Unknown & & 9 \\
\hline Treatment before immunotherapy & 64 & \\
\hline$\geqslant 1$ line of treatment & & $56(87.5)$ \\
\hline Targeted therapy & & $14(21.9)$ \\
\hline \multicolumn{3}{|l|}{ Treatment lines } \\
\hline 0 & & $8(12.5)$ \\
\hline 1 & & $16(25.0)$ \\
\hline 2 & & $21(32.8)$ \\
\hline$\geqslant 3$ & & 19 (29.7) \\
\hline Thoracic radiotherapy & 38 & \\
\hline Yes & & 15 (39.5) \\
\hline No & & $23(60.5)$ \\
\hline Unknown & & 26 \\
\hline Best objective response until ILD diagnosis & 64 & \\
\hline Complete response/partial response & & 23 (35.9) \\
\hline Progression of disease & & $7(10.9)$ \\
\hline Stable disease & & $21(32.8)$ \\
\hline Not evaluated & & 13 (20.3) \\
\hline
\end{tabular}

Data are presented as $\mathrm{n}, \mathrm{n}(\%)$ or median (range). COPD: chronic obstructive pulmonary disease; ECOG: Eastern Cooperative Oncology Group; PD-L: programmed cell death ligand; ILD: interstitial lung disease. 
FIGURE 2 Patients in whom interstitial lung disease developed, stratified by Common Terminology Criteria for Adverse Events ICTCAE; version 4:0). NSCLC: nonsmall cell lung cancer.

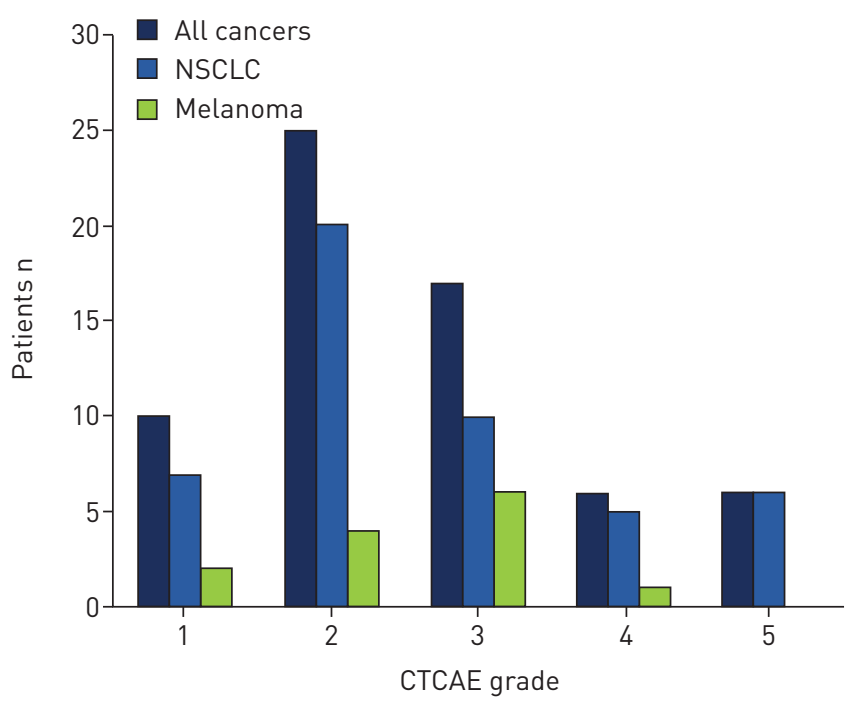

melanoma, respectively. $21(32.8 \%)$ patients had a stable disease and seven (10.9\%) had progressive disease (13 were not evaluated at the time of ILD diagnosis).

\section{Clinical features of ILD}

Severity of ILD was grade 1 in $10(15.6 \%)$ patients, grade 2 in $25(39.1 \%)$ patients, grade 3 in 17 (26.6\%) patients, grade 4 in six (9.4\%) patients and six (9.4\%) cases of ILD were fatal (grade 5), after excluding other causes of death. Among patients with NSCLC, 56.3\% (27 out of 48) experienced grades 1-2 and $43.8 \%$ (21 out of 48) experienced grades 3-5. Among patients with melanoma, 46.2\% (six out of 13) experienced grades 1-2 and 53.8\% (seven out of 13) experienced grades 3-4. In contrast, all grade 5 patients had NSCLC (figure 2). Except for those that were grade 5, no significant difference was observed regarding severity of ILD between cases of NSCLC and melanoma ( $p=0.51)$. The most common symptoms were dyspnoea $(80.3 \%)$ and cough $(52.5 \%)$. Fever was less common $(32.8 \%)$, and four (6.6\%) patients were asymptomatic.

The median time to onset of ILD after introducing immunotherapy was 2.3 (0.2-27.4) months. A majority ( 27 out of $64 ; 42.2 \%$ ) of patients developed ILD $<2$ months after introducing immunotherapy; time to development of ILD was 2-4 months in $26.6 \%$ (17 out of 64 ), $4-6$ months in $17.2 \%$ (11 out of 64 ) and $>6$ months in $14.1 \%$ (nine out of 64 ) of patients (table 2). Onset tended to be earlier in cases of NSCLC (median (range) time to ILD 2.1 (0.2-27.4) months) compared to melanoma (median (range) time to ILD $5.2(0.2-18.1)$ months) ( $p=0.01)$ (figure 3). There was no correlation between median time to onset and severity of ILD, i.e. 2.8 months for grades $1-2$ and 2.2 months for grades $3-5(p=0.32)$. No significant differences were found in the median time to onset between the different types of drugs (anti-CTLA-4, anti-PD1 or anti-PD-L1). No risk factors were identified between patients with grades 1-2 ILD versus grades 3-5 ILD (online supplementary table S3).

\section{Radiological features}

Ground-glass opacities found on a lung CT scan were the most predominant lesions, present in 52 (81.3\%) patients, followed by consolidations in 34 (53.1\%) patients, bronchiectasis in 11 (17.2\%) patients, interlobular septal thickening in $10(15.6 \%)$ patients and intralobular lines in $14(21.9 \%)$ patients. We found two cases of crazy paving. $45(78.9 \%)$ patients had diffuse lung involvement and $12(21.1 \%)$ had localised lung involvement. The median number of lobes involved was three (1-5). We observed asymmetrical topography of lesions in $20.5 \%$ of cases.

Organising pneumonia was the most common pattern (15 out of $64 ; 23.4 \%$ ), followed by hypersensitivity pneumonitis (10 out of $64 ; 15.6 \%$ ). NSIP and bronchiolitis were found in $7.8 \%$ (five out of 64 ) and $6.3 \%$ (four out of 64) of patients, respectively. Six patients had concomitant NSIP and organising pneumonia, and one patient had NSIP plus bronchiolitis (figures 4 and 5 and online supplementary figure S1). It was not possible to describe a suggestive pattern for 23 (35.9\%) patients (table 2). No correlations were observed between time to onset of ILD and radiographic patterns. 


\begin{tabular}{|c|c|}
\hline Delay of occurrence between pneumonitis and introduction of immunotherapy months & $2.3(0.2-27.4)$ \\
\hline $0-2$ & $27(42.2)$ \\
\hline $2-4$ & $17(26.6)$ \\
\hline $4-6$ & $11(17.2)$ \\
\hline$>6$ & $9(14.1)$ \\
\hline \multicolumn{2}{|l|}{ CTCAE grade } \\
\hline 1 & $10(15.6)$ \\
\hline 2 & $25(39.1)$ \\
\hline 3 & $17(26.6)$ \\
\hline 4 & $6(9.4)$ \\
\hline 5 & $6(9.4)$ \\
\hline \multicolumn{2}{|l|}{$\mathrm{BAL}^{\#}$} \\
\hline Yes & $35(55.6)$ \\
\hline No & $28(44.4)$ \\
\hline Unknown & 1 \\
\hline Lymphocytes $\%$ & $33.5(1.0-70.0)$ \\
\hline$\leqslant 15$ & $6(20.0)$ \\
\hline$>15$ & $24(80.0)$ \\
\hline Unknown & 5 \\
\hline \multicolumn{2}{|l|}{ Evolution ${ }^{\#}$} \\
\hline Recovery & $18(28.6)$ \\
\hline Improvement & $25(39.7)$ \\
\hline Stable & $13(20.6)$ \\
\hline Worsening & $1(1.6)$ \\
\hline Fatal & $6(9.5)$ \\
\hline Unknown & 1 \\
\hline \multicolumn{2}{|l|}{ Radiological features } \\
\hline \multicolumn{2}{|l|}{ Lesions } \\
\hline GGO & 52 (81.3) \\
\hline Consolidations & $34(53.1)$ \\
\hline Intralobular lines & $14(21.9)$ \\
\hline Interlobular septal thickening & $10(15.6)$ \\
\hline Traction bronchectasis & $11(17.2)$ \\
\hline Extent (lobes) $n$ & $3(1-5)$ \\
\hline \multicolumn{2}{|l|}{ Pattern } \\
\hline Organising pneumonia & $15(23.4)$ \\
\hline Hypersensitivity pneumonia & $10(15.6)$ \\
\hline NSIP and organising pneumonia & $6(9.4)$ \\
\hline NSIP & $5(7.8)$ \\
\hline Bronchiolitis & $4(6.3)$ \\
\hline NSIP and bronchiolitis & $1(1.6)$ \\
\hline No classification & 23 (35.9) \\
\hline
\end{tabular}

Data are presented as median (range) or $n(\%) . n=64$, unless otherwise stated. CTCAE: Common Toxicity Criteria for Adverse Events, version 4.0; BAL: bronchoalveolar lavage; GGO: ground-glass opacity; NSIP: nonspecific interstitial pneumonitis. ${ }^{\#}: \mathrm{n}=63 ;{ }^{\text {ๆ: }} \mathrm{n}=30$.

\section{BAL and biopsies}

BAL was performed in $35(55.6 \%)$ patients and showed T-lymphocytic alveolitis in 24 (37.5\%) cases. A complete description of the BAL data is shown in online supplementary table S1. Microbiological evaluations (viruses, bacteria, fungi and parasites) were negative. One patient had a positive PCR for Pneumocystis jirovecii in the sputum and received antibiotic treatment, in addition to treatment for ILD. This case was carefully reviewed by the multidisciplinary panel and a diagnosis of ICI-ILD was confirmed because $P$. jirovecii was positive in a PCR of the sputum, but negative for direct examination by standard staining and indirect immunofluorescence, and the radiological data did not favour a diagnosis of $P$. jirovecii pneumonia. Six patients had transbronchial biopsy that showed inflammatory and lymphocytic infiltration (online supplementary figure S2). In addition, PD-L1 tumour expression in patients' tissues was studied using immunohistochemistry (15 samples were available) and was found to be positive in 12 out of 15 patients.

\section{Management}

Corticosteroids and antibiotics were given to $86.9 \%$ and $66.1 \%$ of patients, respectively. ICI was definitively stopped in $76.6 \%$, temporally stopped in $15.6 \%$ and continued in $7.8 \%$. Most patients with 

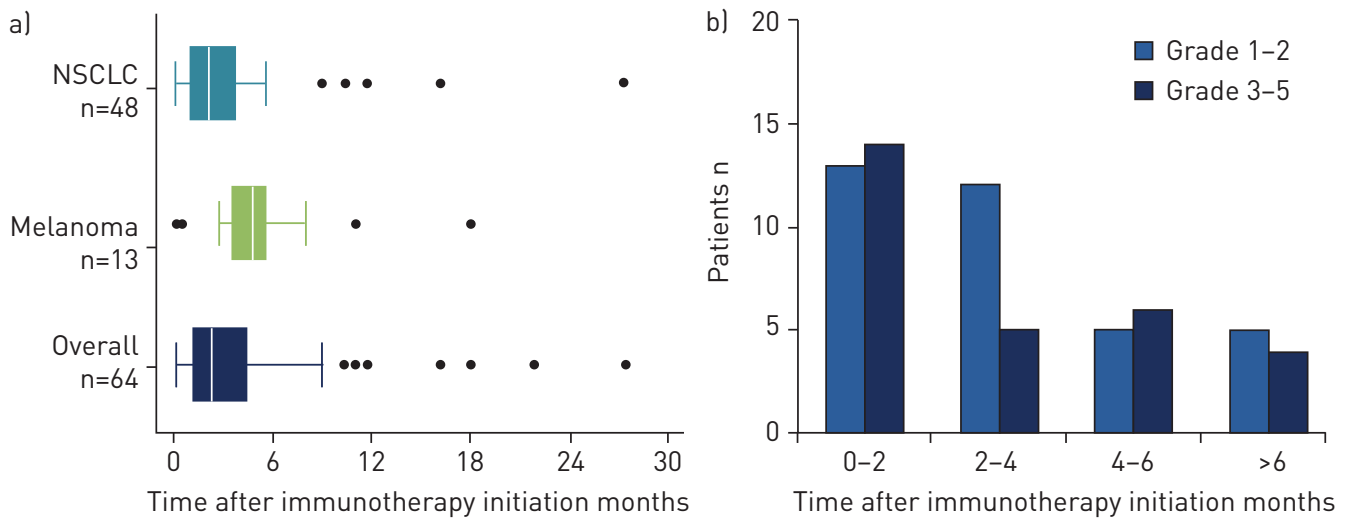

FIGURE 3 a) Time to onset of interstitial lung disease (ILD) stratified by type of cancer. Data are presented as median (interquartile range). Median 2.1 months versus 5.2 months for nonsmall cell lung cancer (NSCLC) and melanoma, respectively; $p<0.02$. b) Time to onset of ILD stratified by severity grade (Common Terminology Criteria for Adverse Events version 4:0).

grade 1 or 2 ILD received oral steroids or drug holding only. Four patients with grade 1 ILD only received drug holding or surveillance with no steroids, and three patients with grade 2 ILD only received drug holding. All patients with grade $\geqslant 3$ ILD received oral or intravenous steroids and drugs were stopped definitively. The median starting dose of prednisone was $80(20-240) \mathrm{mg}$ and the median duration of treatment was $27(4-251)$ days. The median time from ILD diagnosis until initiation of steroids was 2 weeks ( $0-3$ months) and median duration to significant improvement was 0.9 (0.1-7.4) months. In our study, no patient received an immunosuppressive treatment such as infliximab or cyclophosphamide. The maximum immunosuppression used was high-dose prednisone (250 mg per day) (online supplementary table S4).

\section{Clinical outcomes}

During follow-up, 19 (29.7\%) patients died and 45 (70.3\%) patients were still alive. Causes of death included ICI-ILD for six patients; 10 deaths were related to tumour progression; two deaths were from other causes (pulmonary embolism and erosion of pulmonary artery); and one death was caused by Pseudomonas aeruginosa pneumonia.

ILD resolved in 18 (28.6\%) patients and improved in 25 (39.7\%) patients, including 58.1\% (25 out of 43) of patients with grade 1 or 2 ILD and $41.9 \%$ (18 out of 43 ) of patients with grade 3 or 4 ILD. 13 (20.6\%) cases of ILD were stable and one patient was lost to follow-up (table 2). The median time from ILD diagnosis to recovery or significant improvement was 35 (6-239) days.

Fatal ILD occurred exclusively in NSCLC patients treated with anti-PD-1 or PD-L1. Two patients received only one infusion, the four others received between two and eight infusions of PD1/PD-L1 inhibitors. Regarding radiographic patterns, we observed two cases of organising pneumonia, one of hypersensitivity pneumonitis and three cases with no specific pattern. All of these six patients received antibiotics and high-dose steroids $\left(2 \mathrm{mg} \cdot \mathrm{kg}^{-1}\right)$. Overall survival rates at 2, 4 and 6 months were $79.1 \%$ (95\% CI 64.9-88.1), $68.1 \%$ (95\% CI 50.3-80.6) and 58.1\% (95\% CI 37.7-73.8), respectively (figure 6).

\section{Immunotherapy rechallenge}

Immunotherapy was restarted in $10(17.2 \%)$ patients (including one case of melanoma and nine cases of NSCLC) after resolution of ILD. All patients had grade 1-2 ILD and were initially only treated with drug holding or with steroids. Among these 10 patients, recurrent ILD was noted in three cases of NSCLC. Two of these were grade 2 ILD and the third patient had grade 1 ILD. The two patients with an initial grade 2 ILD were treated with drug holding and steroids, and developed recurrent grade-2 ILD after 1 month (two doses) of retreatment. The second episode was successfully treated with drug holding and steroids. For the patient that initially had grade 1 toxicity, the maximum treatment received consisted of drug holding only and an ILD recurred after 4 months (eight doses). Steroids were given as secondary prophylaxis for three patients at 10, 20 and $40 \mathrm{mg}$. One patient had recurrent ILD despite the steroids.

Seven patients did not develop a second ILD event after an ICI rechallenge. Two of these patients died from tumour progression 2 months after the rechallenge (online supplementary table S4) 

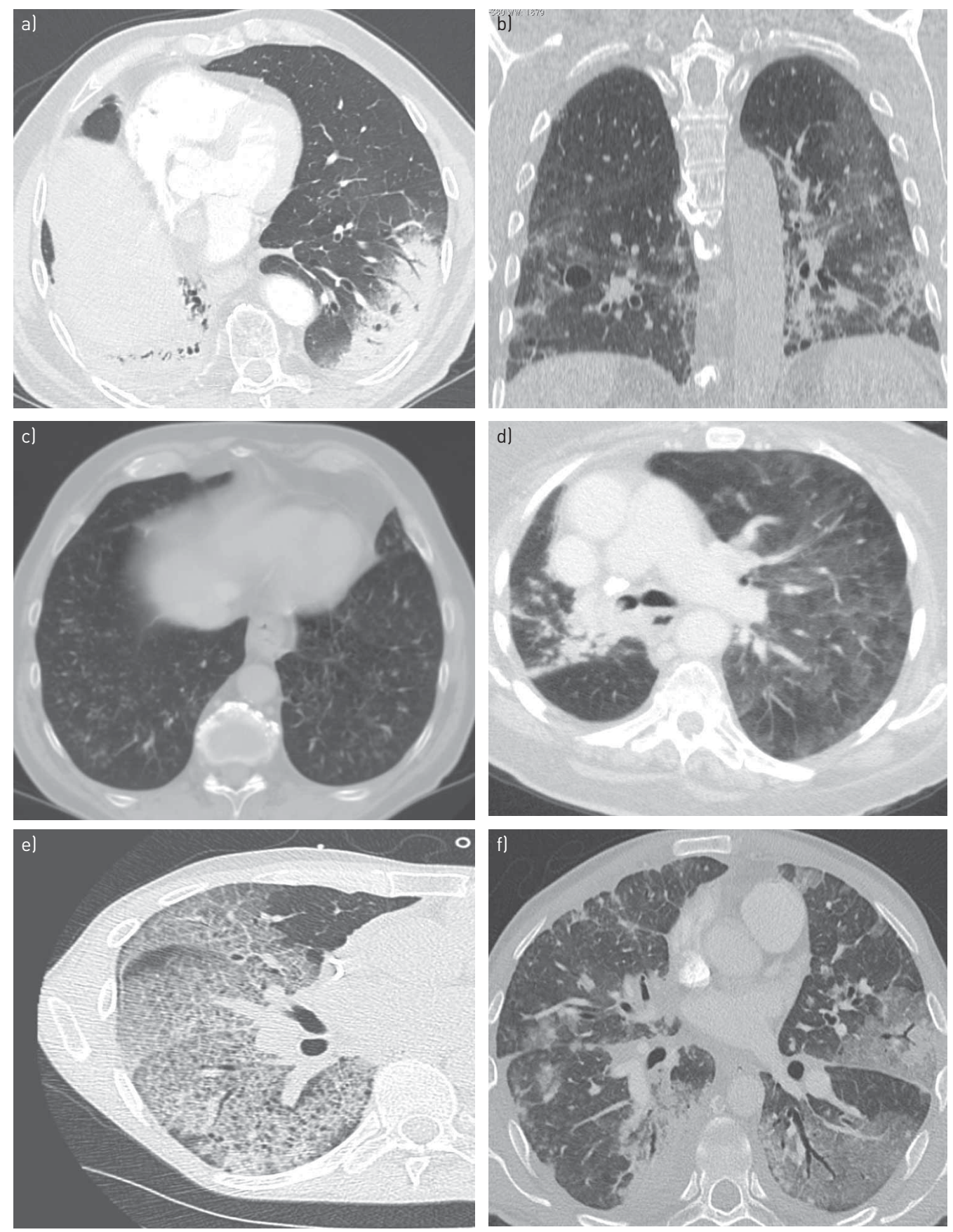

FIGURE 4 Radiological features of immune checkpoint inhibitor-associated interstitial lung disease classified into five patterns. al Organising pneumonia pattern with subpleural consolidations; b) nonspecific interstitial pneumonia pattern: subpleural areas of consolidation with bronchectasis ground-glass opacities and intralobular lines; c) bronchiolitis pattern: low attenuated bilateral centrolobular nodules; d) hypersensitivity pneumonitis pattern: lobular areas of decreased attenuation with mosaic perfusion and ground-glass opacities; el crazy-paving pattern: diffuse ground-glass attenuation with surimposed interlobular septal thickening and intralobular lines; f) no suggestive pattern: diffuse ground-glass opacities in the left upper and lower lobes with interlobular septal thickening and condensation.

\section{Discussion}

We herein report the largest series to date of patients with ICI-ILD. The observation of 64 cases in a very short period of time shows that this side-effect is rare, but not exceptional. ILDs have been reported since the very beginning of clinical trials with ICI. In the CheckMate-017 and - 057 trials, pneumonitis occurred in $4.6 \%$ and $1.4 \%$ of cases of any grade, respectively $[9,18]$. With pembrolizumab, pneumonitis occurred in $5 \%$ of patients in the KEYNOTE-010 trial, and more recently in $5.8 \%$ of the patients in the 
a)

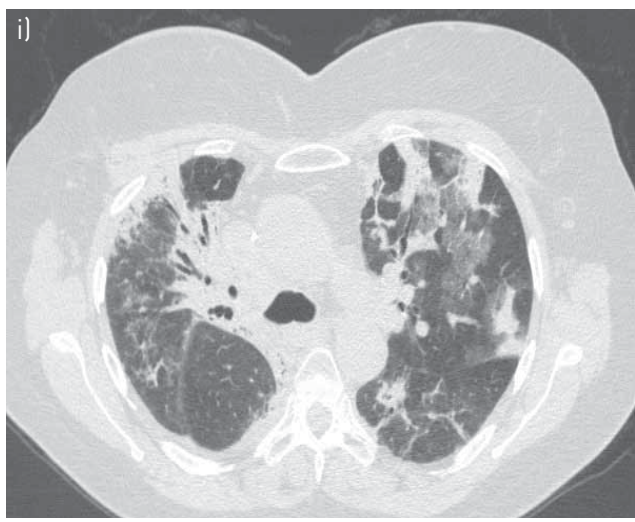

b)

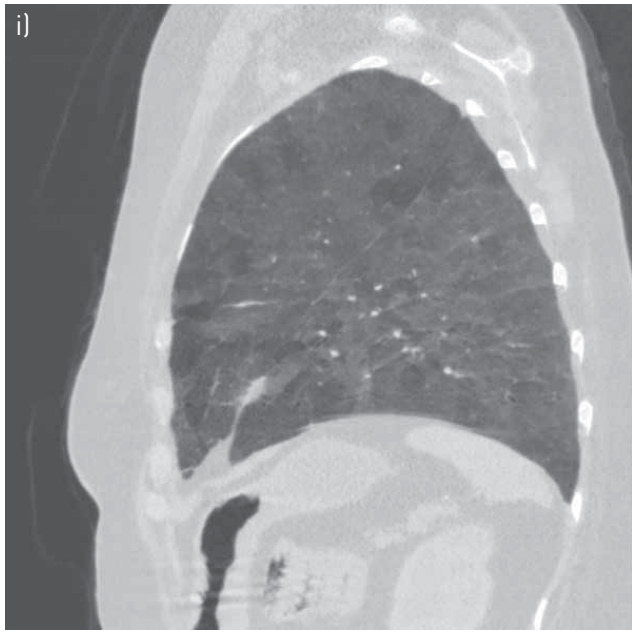

c)

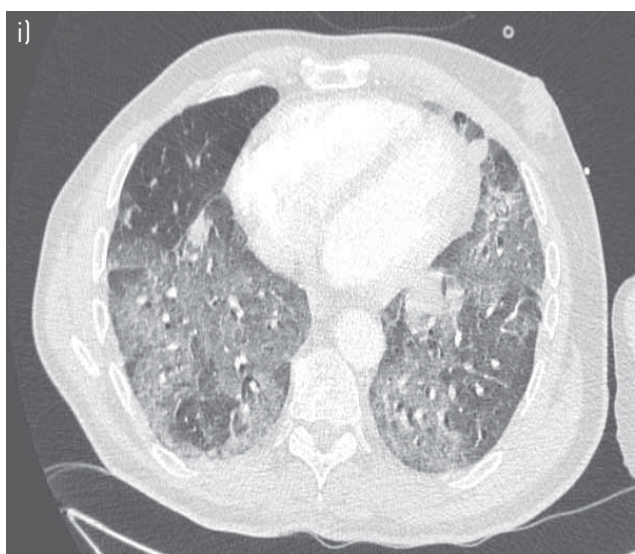

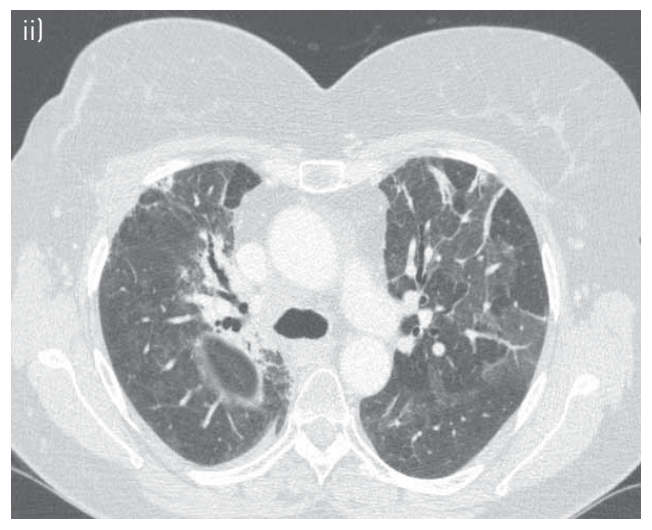
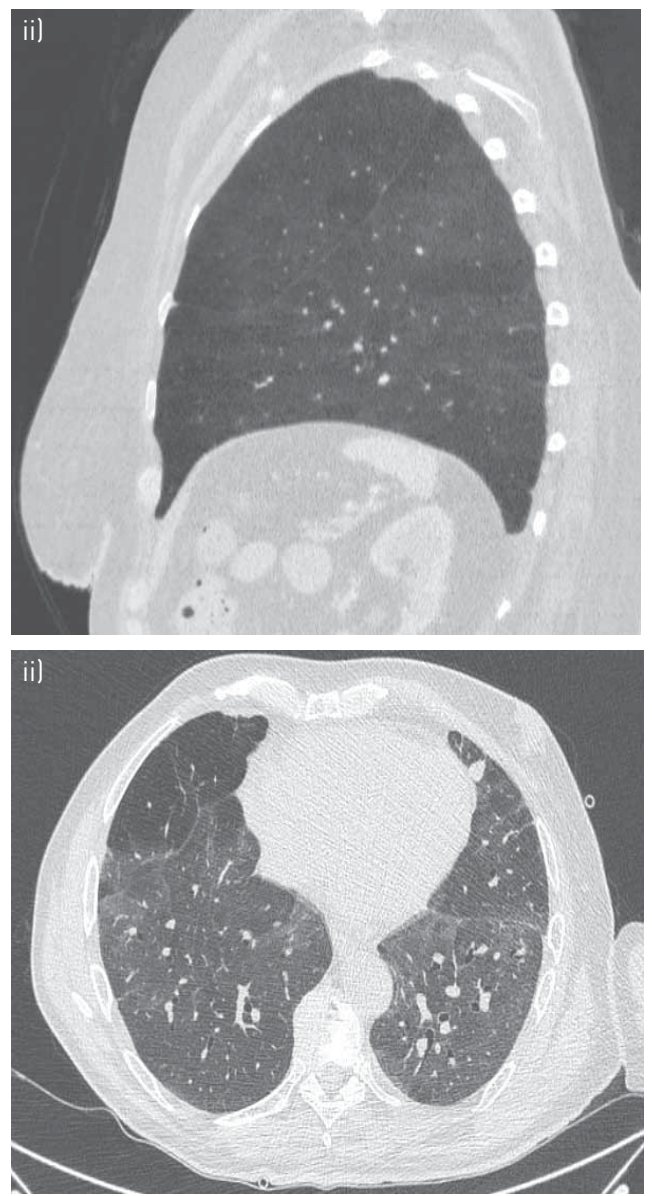

FIGURE 5 Radiological features of immune checkpoint inhibitor (ICI)-associated interstitial lung disease after treatment. a) Organising pneumonia pattern i) at baseline and ii) after 3 months without $\mathrm{ICl}$ and corticosteroids; ground-glass opacities persistent. b) Hypersensitivity pneumonitis pattern (sagittal minimal intensity projection reconstruction) il at baseline and ii) after 6 months without ICl and corticosteroids. c) No suggestive pattern il at baseline and ii) after 1 month without $\mathrm{ICI}$ and corticosteroids.

KEYNOTE-024 trial [1, 19]. A slightly lower incidence was found with PD-L1 inhibitors. Atezolizumab was associated with pneumonitis in $3 \%$ and $1 \%$ of the patients enrolled in the POPLAR and OAK studies, respectively $[5,7]$.

A recent meta-analysis reported an overall incidence of $2.7 \%$ and a $0.8 \%$ occurrence for grade 3 or higher [20]. Two recent studies also reported a higher incidence (5\% and $11.8 \%$, respectively) [10, 11]. In our series the overall incidence was estimated at $3.5 \%$. Consequently, we anticipate that, due to the large diffusion of these treatments, most clinicians will have to deal with this side-effect. ICI-ILD appears to be 
FIGURE 6 Overall survival of the global population of patients with interstitial lung disease (ILD). Calculated from the time of ILD diagnosis to the first event: death or date of last news.

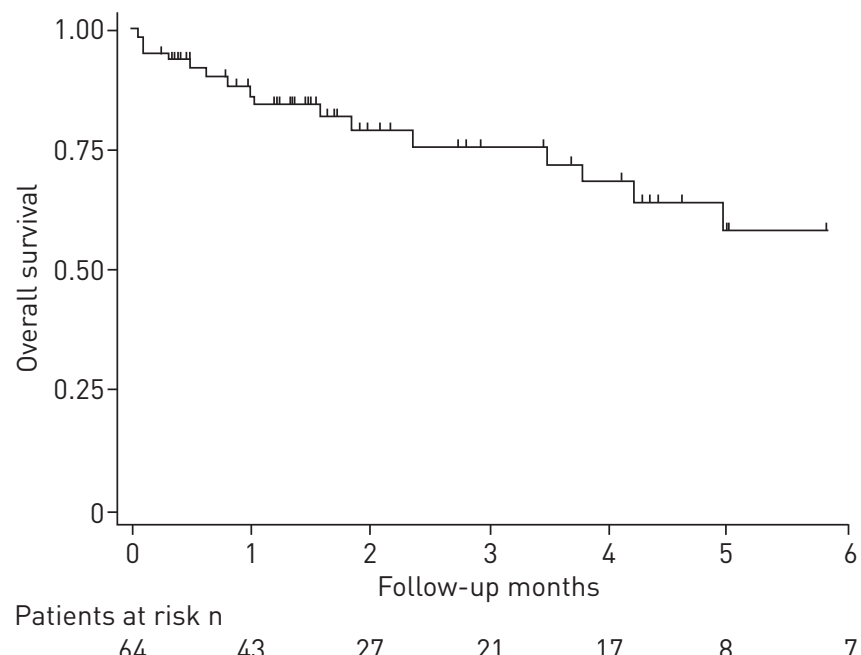

more frequent than is observed with other drugs used to treat NSCLC, such as pemetrexed, erlotinib, gefitinib, docetaxel, gemcitabine or crizotinib [21-27].

A diagnosis of ICI-ILD is not straightforward, as the symptoms are not specific. Clinicians should maintain a high degree of suspicion in cases that manifest new respiratory symptoms while receiving ICI. We showed that most of these cases of ILD occurred during the first months of treatment (median time to onset 2.3 months). Two studies have also reported early onset (2.8 months and 2.6 months) [10, 11]. In our series, the time to onset tended to be earlier in cases of NSCLC than for melanoma. Furthermore, ILD-related death was noted only in patients with NSCLC. These differences between NSCLC and melanoma could be caused by exposure to tobacco smoke and the underlying lung status. However, our finding must be interpreted with caution due to the differences between these populations (48 versus 13 patients). In a meta-analysis, the incidence of ILD remained significantly higher for NSCLC compared to melanoma, adding evidence for this hypothesis [20].

We found a high frequency of radiological patterns such as organising pneumonia or hypersensitivity pneumonitis, and some cases of NSIP. Previous reports have already documented organising pneumonia and NSIP [28-30].

To minimise the incidence of this toxicity, it will be beneficial to identify patients at higher risk in order to exclude them from this therapeutic strategy or to propose a closer follow-up. Parameters such as COPD or a history of radiotherapy are known to be associated with a higher risk of pneumonitis in patients treated with EGFR and tyrosine kinase inhibitors [31]. As our series was retrospective and did not have a comparative group, we were not able to highlight the risk factors. Nevertheless, the high number of smokers, patients with COPD or with a history of radiotherapy needs to be further analysed. PD-L1 expression is often found in these patients, but further studies are needed to accurately predict potential lung toxicities.

There is no validated recommendation for ICI-ILD management, which is usually guided by clinical experience and observational reports [32, 33]. Progressive cancer lesions, infection and pulmonary embolism are frequent complications seen in lung cancers and other tumours. Thus, we highly recommend performing a CT scan with a contrast agent to eliminate differential diagnoses and to provide evidence for ICI-ILD. Moreover, we think that bronchoscopy with BAL plus distal biopsies can help eliminate the presence of infections or tumour progression, and provide clues regarding immune-related pneumonitis, such as a high percentage of lymphocytes and their associated histological characteristics.

The treatment of ICI-ILD is based on systemic steroids. For grade 1-2 ILD, drug holding and only CT monitoring after $2 / 3$ weeks can be considered. If a course of steroids does not reduce the severity of the initial symptoms, additional immunosuppression could be considered. The infectious risk and lung toxicity of anti-tumour necrosis factor (TNF) and the lack of knowledge about the physiopathological mechanisms of ICI-ILD make the use of this biotherapy particularly complex. Its use must be discussed by a multidisciplinary board. In our series, most patients had a favourable outcome when treated with steroids. No anti-TNF- $\alpha$ drugs were given.

The mechanisms of ILD related to checkpoint inhibitors are still undetermined, but we can hypothesise that they involve deregulation of immune effectors and T-cells in the pulmonary interstitium, leading to a 
subsequent inflammatory response. Moreover, a predominance of lymphocytes was found upon BAL and biopsies showed infiltration of lymphocytes. Additionally, an increase in the number of activated T-cells in the BAL fluid of patients with organising pneumonia has been reported when compared to normal control subjects, suggesting that T-cells may play an important role in the pathogenesis of this disease [34]. The high lymphocyte counts in BAL fluid and the rapid clinical improvement seen with administration of steroids or treatment withdrawal suggests an immune-mediated mechanism of lung injury [35]. Some authors describe sarcoid-like granulomas induced by ipilimumab or nivolumab. In our series we did not encounter cases of sarcoid-like granulomatosis, but BAL and transbronchial biopsies were not performed systematically, and so may have underestimated the incidence of sarcoid-like granulomatosis diagnosis [36].

PD-L1 inhibitors are supposed to be less toxic than PD1 inhibitors because they do not prevent interactions between PDL-2 and PD1, but we cannot confirm this apparent difference in our study, as most patients were treated with PD1 inhibitors. We cannot firmly conclude about PD-L1 expression, because we only had 15 biopsies; however, some authors suggest that decreased expression of PD-L1 can contribute to unregulated local inflammation and dermatological toxicities, such as psoriatic epidermis [37]. Another hypothesis is that PD1 inhibition increases the interaction between RGMb (repulsive guidance protein b) and PD-L2 by reducing interactions between PD1 and PD-L2, which may result in a clonal expansion of lung-resident T-cells and a decrease in the immune-tolerance signalling pathways [38].

A further issue yet to be determined is whether immune-related adverse events can be correlated with efficacy. Retrospective studies found higher rates of disease control in patients who experienced immune-related adverse events $[39,40]$. In our series, overall survival was $\sim 6$ months and the response at the time of pneumonitis was $35.6 \%$, which is better than expected in this highly pretreated population with advanced-stage disease. However, our series is too limited to determine whether ILD was associated with a good outcome in nonfatal cases. It also remains debatable whether severity of toxicity can be used as a marker for a response, as observed with some targeted therapies [27]. A potential bias is that side-effects occur more frequently in patients receiving "more" drugs and are thus more likely to respond.

In conclusion, our large cohort provides new insights into the diagnosis and treatment of ICI-ILD. We have shown that this toxicity is not exceptional, that it usually occurs during the first months and that it associated with nonspecific clinical symptoms and suggestive radiological signs. BAL and bronchial biopsies can refine a diagnosis by excluding pulmonary infection and showing lymphocytic alveolitis. Awareness of the radiographic and clinical manifestations of ICI-ILD is critical to obtain a prompt diagnosis and to manage this potentially serious adverse event.

\section{References}

1 Reck M, Rodríguez-Abreu D, Robinson AG, et al. Pembrolizumab versus chemotherapy for PD-L1-positive non-small-cell lung cancer. N Engl J Med 2016; 375; 1823-1833.

2 Robert C, Long GV, Brady B, et al. Nivolumab in previously untreated melanoma without BRAF mutation. $N$ Engl J Med 2015; 372: 320-330.

3 Robert C, Schachter J, Long GV, et al. Pembrolizumab versus ipilimumab in advanced melanoma. N Engl J Med 2015; 372: 2521-2532.

4 Ansell SM, Lesokhin AM, Borrello I, et al. PD-1 blockade with nivolumab in relapsed or refractory Hodgkin's lymphoma. N Engl J Med 2015; 372: 311-319.

5 Rosenberg JE, Hoffman-Censits J, Powles T, et al. Atezolizumab in patients with locally advanced and metastatic urothelial carcinoma who have progressed following treatment with platinum-based chemotherapy: a single-arm, multicentre, phase 2 trial. Lancet 2016; 387: 1909-1920.

6 Ferris RL, Blumenschein G Jr, Fayette J, et al. Nivolumab for recurrent squamous-cell carcinoma of the head and neck. N Engl J Med 2016; 375: 1856-1867.

7 Fehrenbacher L, Spira A, Ballinger M, et al. Atezolizumab versus docetaxel for patients with previously treated non-small-cell lung cancer (POPLAR): a multicentre, open-label, phase 2 randomised controlled trial. Lancet 2016; 387: 1837-1846.

8 Herbst RS, Baas P, Kim DW, et al. Pembrolizumab versus docetaxel for previously treated, PD-L1-positive, advanced non-small-cell lung cancer (KEYNOTE-010): a randomised controlled trial. Lancet 2016; 387: 1540-1550.

9 Brahmer J, Reckamp KL, Baas P, et al. Nivolumab versus docetaxel in advanced squamous-cell non-small-cell lung cancer. N Engl J Med 2015; 373: 123-135.

10 Nishino M, Ramaiya NH, Awad MM, et al. PD-1 inhibitor-related pneumonitis in advanced cancer patients: radiographic patterns and clinical course. Clin Cancer Res 2016; 22: 6051-6060.

11 Naidoo J, Wang X, Woo KM, et al. Pneumonitis in patients treated with anti-programmed death-1/programmed death ligand 1 therapy. J Clin Oncol 2017; 35: 709-717.

12 Camus P, Fanton A, Bonniaud P, et al. Interstitial lung disease induced by drugs and radiation. Respiration 2004; 71: 301-326.

13 Sakai $\mathrm{F}$, Johkoh $\mathrm{T}$, Kusumoto $\mathrm{M}$, et al. Drug-induced interstitial lung disease in molecular targeted therapies: high-resolution CT findings. Int J Clin Oncol 2012; 17: 542-550. 
14 US Department of Health and Human Services, National Institutes of Health, National Cancer Institute. Common Terminology Criteria for Adverse Events (CTCAE) Version 4.0. NIH Publication No 09-5410 2010: May 28, 2009 (v4.03: June 14, 2010).

15 Mueller-Mang C, Grosse C, Schmid K, et al. What every radiologist should know about idiopathic interstitial pneumonias. Radiographics 2007; 27: 595-615.

16 Travis WD, Costabel U, Hansell DM, et al. An official American Thoracic Society/European Respiratory Society statement: update of the international multidisciplinary classification of the idiopathic interstitial pneumonias. Am J Respir Crit Care Med 2013; 188: 733-748.

17 Eisenhauer EA, Therasse P, Bogaerts J, et al. New response evaluation criteria in solid tumours: revised RECIST guideline (version 1.1). Eur J Cancer 2009; 45: 228-247.

18 Borghaei H, Paz-Ares L, Horn L, et al. Nivolumab versus docetaxel in advanced nonsquamous non-small-cell lung cancer. $N$ Engl J Med 2015; 373: 1627-1639.

19 Herbst RS, Baas P, Kim DW, et al. Pembrolizumab versus docetaxel for previously treated, PD-L1-positive, advanced non-small-cell lung cancer (KEYNOTE-010): a randomised controlled trial. Lancet 2016; 387: $1540-1550$

20 Nishino M, Giobbie-Hurder A, Hatabu $\mathrm{H}$, et al. Incidence of programmed cell death 1 inhibitor-related pneumonitis in patients with advanced cancer: a systematic review and meta-analysis. JAMA Oncol 2016; 2 : 1607-1616.

21 Liu V, White DA, Zakowski MF, et al. Pulmonary toxicity associated with erlotinib. Chest 2007; 132 : $1042-1044$.

22 Konishi J, Yamazaki K, Kinoshita I, et al. Analysis of the response and toxicity to gefitinib of non-small cell lung cancer. Anticancer Res 2005; 25: 435-441.

23 Grande C, Villanueva MJ, Huidobro G, et al. Docetaxel-induced interstitial pneumonitis following non-small-cell lung cancer treatment. Clin Transl Oncol 2007; 9: 578-581.

24 Roychowdhury DF, Cassidy CA, Peterson P, et al. A report on serious pulmonary toxicity associated with gemcitabine-based therapy. Invest New Drugs 2002; 20: 311-315.

25 Hochstrasser A, Benz G, Joerger M, et al. Interstitial pneumonitis after treatment with pemetrexed: a rare event? Chemotherapy 2012; 58: 84-88.

26 Loriot Y, Ferte C, Gomez-Roca C, et al. Pemetrexed-induced pneumonitis: a case report. Clin Lung Cancer 2009; 10: $364-366$

27 Créquit P, Wislez M, Fleury Feith J, et al. Crizotinib associated with ground-glass opacity predominant pattern interstitial lung disease: a retrospective observational cohort study with a systematic literature review. $J$ Thorac Oncol 2015; 10: 1148-1155.

28 Nishino M, Sholl LM, Hodi FS, et al. Anti-PD-1-related pneumonitis during cancer immunotherapy. $N$ Engl $J$ Med 2015; 373: 288-290.

29 Nakashima K, Naito T, Omori S, et al. Organizing pneumonia induced by nivolumab in a patient with metastatic melanoma. J Thorac Oncol 2016; 11: 432-433.

30 Gounant V, Brosseau S, Naltet C, et al. Nivolumab-induced organizing pneumonitis in a patient with lung sarcomatoid carcinoma. Lung Cancer 2016; 99: 162-165.

31 Chiang CL, Chen YW, Wu MH, et al. Radiation recall pneumonitis induced by epidermal growth factor receptor-tyrosine kinase inhibitor in patients with advanced nonsmall-cell lung cancer. J Chin Med Assoc 2016; 79: $248-255$.

32 Lynch TJ, Bondarenko I, Luft A, et al. Ipilimumab in combination with paclitaxel and carboplatin as first-line treatment in stage IIIB/IV non-small-cell lung cancer: results from a randomized, double-blind, multicenter phase II study. J Clin Oncol 2012; 30: 2046-2054.

33 Inoue A, Kunitoh H, Sekine I, et al. Radiation pneumonitis in lung cancer patients: a retrospective study of risk factors and the long-term prognosis. Int J Radiat Oncol Biol Phys 2001; 49: 649-655.

34 Barjaktarevic IZ, Qadir N, Suri A, et al. Organizing pneumonia as a side effect of ipilimumab treatment of melanoma. Chest 2013; 143: 858-861.

35 Satake N, Nagai S, Kawatani A, et al. Density of phenotypic markers on BAL T-lymphocytes in hypersensitivity pneumonitis, pulmonary sarcoidosis and bronchiolitis obliterans with organizing pneumonia. Eur Respir J 1993; 6: 477-482.

36 Berthod G, Lazor R, Letovanec I, et al. Pulmonary sarcoid-like granulomatosis induced by ipilimumab. J Clin Oncol 2012; 30: e156-e159.

37 Kim DS, Je JH, Kim SH, et al. Programmed death-ligand 1, 2 expressions are decreased in the psoriatic epidermis. Arch Dermatol Res 2015; 307: 531-538.

38 Tabchi S, Messier C, Blais N. Immune-mediated respiratory adverse events of checkpoint inhibitors. Curr Opin Oncol 2016; 28: 269-277.

39 Bronstein Y, Ng CS, Hwu P, et al. Radiologic manifestations of immune-related adverse events in patients with metastatic melanoma undergoing anti-CTLA-4 antibody therapy. AJR Am J Roentgenol 2011; 197: W992-W1000.

40 Osorio JC, Ni A, Chaft JE, et al. Antibody-mediated thyroid dysfunction during T-cell checkpoint blockade in patients with non-small-cell lung cancer. Ann Oncol 2017; 28: 583-589. 